\title{
Testing environmental influences on star formation with a sample of Low Surface Brightness dwarf galaxies in the Vigo cluster
}

\author{
Ana Heller, Elchanan Almoznino \& Noah Brosch \\ The Wise Observatory and the School of Physics and Astronomy \\ Tel Aviv University, Tel Aviv 69978, Israel
}

\begin{abstract}
We analyze the star formation activity of an homogeneous LSB dwarf galaxy sample in the Virgo cluster, as a function of the radial velocity relative to the cluster mean velocity and the projected distance from the center of the cluster, using CCD images obtained at the Wise Observatory. The localized $\mathrm{H} \alpha$ emission in the HII regions of this sample is compared to that of an isolated gas-rich sample of LSB dwarf galaxies and that of a representative sample of Blue Compact Dwarf (BCD) galaxies in the cluster. We report preliminary results on the LSB dwarf star formation histories obtained from surface color distribution.
\end{abstract}

Keywords: LSB dwarf galaxies, environment, star formation

\section{Introduction}

There is an endless debate in the literature regarding the influence of the neighborhood over the physical processes that govern the evolution and the relative abundance of types in dwarf galaxies (Koopmann 1997). Futhermore, recent investigations of distant galaxies suggest that while rich cluster galaxies have reduced star formation compared to field galaxies of the same central concentration of light, the star formation rate (SFR) is quite sensitive to local galaxy density, both inside and outside of clusters, but the highest levels of star formation are encountered in the intermediate density environment (Hashimoto et al. 1997). Therefore, we must consider not only the surface brightness of galaxies in each class, but also their environments, to see if they influence the star formation properties.

The high Galactic latitude of the Virgo cluster (VC), and therefore very small foreground extinction, coupled with the relative nearness of the cluster, allows one to study the effect of the environment on the star formation histories of low surface brightness (LSB) dwarf galaxies. Their low mean density and gravitational binding energies (Bothun et al. 1985) make them susceptible to dynamical processes that potentially operate in the cluster, which has a mean galaxy density $\sim 10$ galaxies $\mathrm{Mpc}^{-3}$. However, the $\mathrm{VC}$ is not a relaxed, virialized cluster; it shows a complex structure in which the LSB dwarf population has no preferential velocity distribution. A kinematic structure map, showing the position on the sky of the various groups and clouds within the VC cluster area, has been published by Hoffman et al. (1989). 
External forces produced by tidal interactions or tidal shocks should depend strongly on the mass of the perturber/perturbed galaxy, the relative speed, and the minimal distance between them. Other effects of the surroundings, such as ram pressure stripping, evaporation, or turbulent viscous stripping, are believed to be active in gas removal, with estimated stripping time-scale $\sim 10^{9} \mathrm{yr}$, and should be most efficient in the hot and dense central part of the VC, within $\sim 300 \mathrm{kpc}$ of M87 (Ferguson \& Binggeli 1994). The removal of gas by the cluster environment is also believed to be responsible for the dependence of chemical abundance properties of HII regions on cluster location in late-type spiral galaxies in VC (Skillman et al. 1995). Note that none of the LSB dwarf galaxies included in our sample (see below) is located in the central region of the $\mathrm{VC}$, and all of them are more distant than $\sim 2.5$ degrees ( $\sim 1 \mathrm{Mpc}$, projected) from M87. Therefore, the processes mentioned before may not be affecting these galaxies. This is supported by VLA HI maps of the more intense sources of our sample of galaxies (Skillman \& Bothun 1986, Skillman et al. 1987)) and Arecibo HI maps (Hoffman et al. 1996, Salpeter \& Hoffman 1996). We found that all mapped galaxies support the previous finding of Skillman et al. (1987); at specific projected distances from the cluster center $\left(\mathrm{R}_{M 87}\right)$, the derived $\mathrm{HI}$ to optical diameter ratios $\left(\mathrm{D}_{H I} / \mathrm{D}_{\text {opt }}\right)$ are larger than those of a sample of $\mathrm{VC}$ spiral galaxies.

However, long before passing near the cluster core region, the pressure of the intracluster medium (ICM) may induce star formation in a gas-rich galaxy, due to processes such as compression of gas clouds, density enhancements, accumulation of gas in clouds that later collapse gravitationally, or cloud-cloud collisions in the interstellar medium (ISM) (Elmegreen 1997). Pressure confinement may also prevent or reduce the outflow of gas driven by supernovae or winds from OB stars (Babul \& Rees 1992). If these dynamical effects are capable to significantly drive the star population and evolution of LSB dwarf galaxies in the $\mathrm{VC}$, we should then expect to see systematic differences in their SFR related to the location within the cluster.

\section{The sample}

In the Virgo Cluster Catalog (VCC, Binggeli, Sandage \& Tammann 1985), the surface brightness serves as a luminosity class indicator for late-type galaxies. The highest surface brightness objects are assigned to class III while those with the lowest surface brightness belong to class $V$. There are 31 galaxies in the VCC with certain classification ImIV and $\operatorname{ImV}$; these are LSB galaxies with mean surface brightness fainter than 24-25 mag arcsec ${ }^{-2}$. We rejected two galaxies from the $24 \mathrm{ImIV}$ to $\operatorname{ImV}$ galaxies with non-zero HI measurements (Hoffman et al. 1987): a small, faint 17.5 magnitude galaxy which fell below our threshold, and another with very high heliocentric radial velocity, which violated our membership $\mathrm{VC}$ criterion of $v_{\odot}<3000 \mathrm{~km} \mathrm{sec}{ }^{-1}$. This $v_{\odot}$ restriction arises because of the void behind the $\mathrm{VC}$, between the $\mathrm{W}$ and $\mathrm{M}$ clouds, from $2800 \mathrm{~km} / \mathrm{sec}$ to $3500 \mathrm{~km} / \mathrm{sec}$, where no galaxies are detected (Binggeli et al. 1993). Here we consider all members in the various clouds as part of the VC. The mean heliocentric radial velocities of the galaxies is $1200 \mathrm{~km} / \mathrm{sec}$, close to the mean 
velocity of $\mathrm{dE}$ galaxies, $1139 \pm 67 \mathrm{~km} / \mathrm{sec}$, though slightly higher than the mean velocity of the cluster $(1050 \pm 35 \mathrm{~km} / \mathrm{sec}$; Binggeli et al. 1993).

Our final sample sample comprises of 27 galaxies; it includes 22 galaxies of type $\operatorname{ImIV}$ to $\operatorname{Im} V$, four of uncertain classification $\operatorname{ImV} / \mathrm{dE}, \operatorname{Im} V$ ? or $\operatorname{Im}$;, and one Im III-IV pec. The limiting magnitude for inclusion in our sample is $m_{B}=17.5$; the galaxies are small and their major-axes range from 16 to $120 \operatorname{arcsec}$ at the 25 mag $\operatorname{arcsec}^{-2}$ isophote.

\section{Observations, Results and Analysis}

In order to study the on-going star formation we observed the galaxies with a narrow-band filter in the red continuum near $\mathrm{H} \alpha$ (H $\alpha$-off) and with a set of narrow-band filters centered on the rest-frame $\mathrm{H} \alpha$ line ( $\mathrm{H} \alpha$-on) of the galaxies. Deep images in the broad-band filters $U, B, V, R$ and I provided some constraints on the older stellar populations. The narrow-band $\mathrm{H} \alpha$ images were taken during the observing runs of 1996 and 1997 at the Wise Observatory (WO). Two of the faintest galaxies were observed with narrow-band $\mathrm{H} \alpha$ filters at the SAO $6 \mathrm{~m}$ telescope. The $\mathrm{H} \alpha$-on images were calibrated with observations of spectrophotometric standards, co-added, and the $\mathrm{H} \alpha$-off image was subtracted from each one to produce final net- $\mathrm{H} \alpha$ images. The $\mathrm{H} \alpha$ images of the sampled galaxies are shown in Heller et al. (1998). The typical limiting $\mathrm{H} \alpha$ flux is $10^{-16}$ erg $\mathrm{sec}^{-1} \mathrm{~cm}^{-2}$; the limiting spatial resolution is $300-400 \mathrm{pc}$. All UBVRI images were collected at the WO during 1997 and 1998 and standard stars (Landolt 1973,1992 ) were used for calibration.

We detected $\mathrm{H} \alpha$ emission in $62 \%$ of the sample galaxies. The detection rate is higher when considering only those galaxies with certain $\operatorname{ImIV}$ to $\operatorname{ImV}$ classification (68\%). Three of the galaxies in which we did not detect $\mathrm{H} \alpha$ emission were those with uncertain classification (dE2 or ImIV, Im?) in the catalog. Their HI fluxes put them in low HI content group with a line flux integral $\leq 776$ $\mathrm{mJy} \mathrm{Km} / \mathrm{sec}$. The high $\mathrm{HI}$ content group, with an equal number of galaxies, had a line flux integral $\geq 950 \mathrm{mJy} \mathrm{Km} / \mathrm{sec}$.

We calculated the total SFR of the galaxies as in Kennicutt et al. (1994), $\mathrm{SFR}=2.9310^{11} \mathrm{~F}(\mathrm{H} \alpha)$, where $\mathrm{F}(\mathrm{H} \alpha)$ is the total line flux in cgs units and the $\mathrm{SFR}$ is in $\mathrm{M}_{\odot} \mathrm{yr}^{-1}$. We adopted a common distance of $18 \mathrm{Mpc}$ for all the galaxies of our sample. This may not be exactly true, but allows a comparison with the values calculated in the same way for BCD galaxies in the VC (Almoznino 1996). The typical SFR of our LSB sample is $0.007 \mathrm{M}_{\odot} \mathrm{yr}^{-1}$ a factor 10 lower than BCDs. The typical $\mathrm{H} \alpha$ equivalent width (EW) of the LSB galaxies is $\sim 30 \AA$ peaking at $100 \AA$, a factor 2 lower than the BCDs.

Some of the LSB galaxies in the sample have low heliocentric velocities; it is possible that these are objects falling into the $\mathrm{VC}$ from its distant side (Tully \& Shaya 1984). We tested the possibility that these galaxies may have enhanced star formation because of interaction with the cluster gas, but this did not prove out. Likewise, we did not find any clear dependence between the net- $\mathrm{H} \alpha$ emission, the $\mathrm{EW}$, the relative velocity of a galaxy with respect to the mean cluster velocity, the angular distance to the $\mathrm{VC}$ center, subclustering, or the HI flux of a galaxy. 
A number of tests were performed on the level of individual HII regions. We first compared the range of net- $\mathrm{H} \alpha$ fluxes of individual HII regions of our LSB sample to those of isolated, gas rich LSB galaxies studied by van Zee (1998) and found that they are extremely similar (Figure 1).

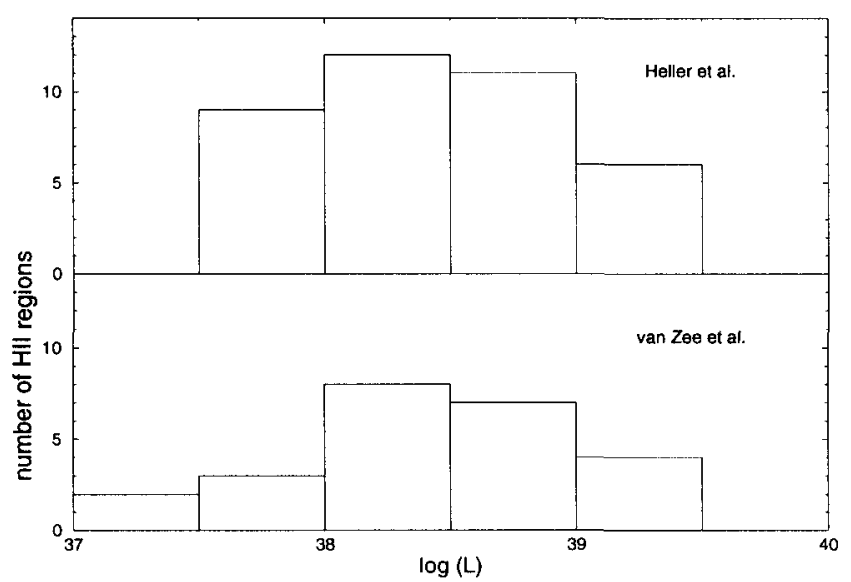

Figure 1. Distribution of the logarithm of the luminosity for individual HII regions, Virgo Cluster LSB dwarf sample (Heller et al. 1998) (up), isolated gas rich LSB sample (van Zee 1998) (down).

Both samples have approximately the same number of HII regions per galaxy and in both the luminosity peaks at $\sim 310^{39} \mathrm{erg} \mathrm{s}^{-1}$. The results indicate no differences between galaxies in the cluster and isolated ones as long as they are dwarfs LSBs, although it should be noted that the covering factor of a galaxy by HII regions is much lower in the isolated sample, originally selected to have extended $\mathrm{HI}$ envelopes.

In Figure 2 we plotted the line and continuum fluxes of the HII regions of each galaxy, including the HII regions of the BCD sample. The dotted lines indicate $\mathrm{EW}=1000,100,10$ and $1 \AA$ respectively. The dearth of HII regions with low EW is easy to understand. It is the result of our detection technique, where low EW HII regions can hardly be distinguished against the red continuum background. On the other hand, it is not clear what limits the high end of the EW distribution. It is very interesting to note that both samples align with approximately the same EW for their HII regions between $\mathrm{EW}=10$ and $100 \AA$.

In a model in which the interaction between the ISM and the ICM is the dominant star formation trigger, the spin of the galaxy moves the HII region from the recent SF burst, which is the locus of the interaction with the ICM, away from the galaxy leading edge. As a burst evolves, the $\mathrm{H} \alpha \mathrm{EW}$ should decrease. This is because of the reduction of the line emission (a few Myrs after the disappearance of the ionizing flux) at the same time as the continuum increases due to the net increase in the number of low mass stars. Note that this is true for a star burst which takes place in a pre-existing old population. Our results for individual HII regions in each galaxy (Figure 3) do not support this scenario. 


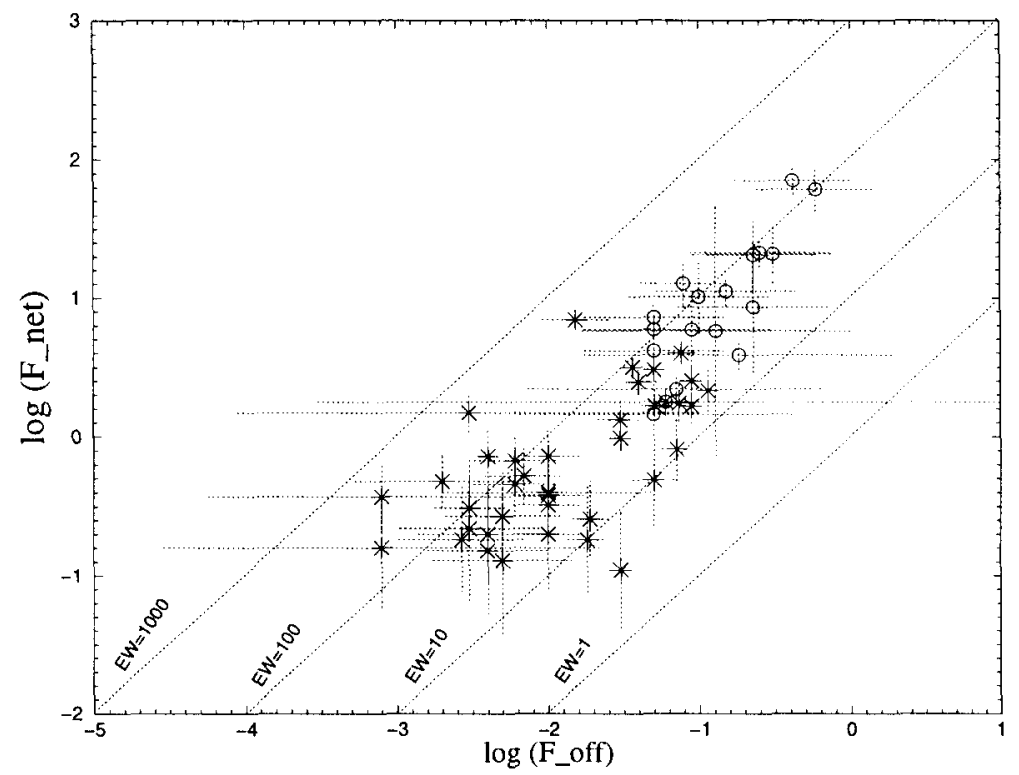

Figure 2. Correlation between the logarithms of the $\mathrm{H} \alpha$ emission and of the red continuum light for individual HII regions in LSB galaxies. The data for HII regions of BCDs is plotted as circles and that for the HII regions of LSBs is represented by stars. The units are $10^{-14}$ erg $\mathrm{cm}^{-2} \mathrm{~s}^{-1}$ for the line flux and $10^{-14} \mathrm{erg} \mathrm{cm}^{-2} \mathrm{~s}^{-1} \AA^{-1}$ for the continuum flux density. The dotted lines indicate $\mathrm{EW}=1000,100,10$ and $1 \AA$ respectively.

An analysis of the number distribution of the star formation regions for these galaxies (and in other star-forming dwarfs), based on the visual inspection of the net- $\mathrm{H} \alpha$ images, results in asymmetric indices ( $A I$, ratio of the number of HII regions counted in the poor to the rich area) $A I<0.5$ (Brosch et al. 1998a and these proceedings). In other words, the HII regions are located predominantly on one side of the galaxy, but are not the result of a bow-shock induced SF.

In case that the bursts are the result of both external and internal agents, we should expect to see a broad range of EW values due to the different locations of the orbiting galaxies in the cluster. For most of the objects this is not the case (Figure 4), and the EW of individual HII regions changes very smoothly in a galaxy, with no correlation with the angular distance to the cluster center (M87) nor with the heliocentric radial velocity of the galaxy.

Lacking a clear understanding of external triggers which may be relevant to explain the star formation in Virgo LSB dwarfs, we turned to internal effects which might influence this process. We searched for correlations between the SFR and other individual observable parameters of the galaxies in the sample. 

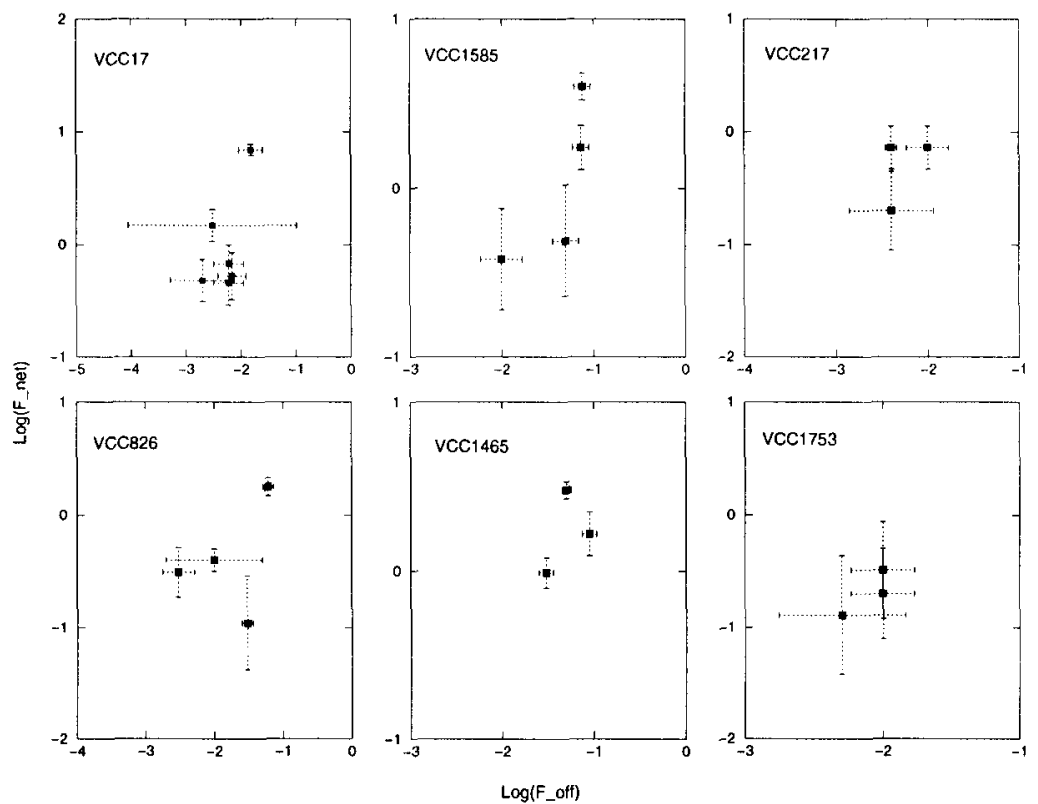

Figure 3. Correlation between the logarithms of the $\mathrm{H} \alpha$ emission and of the red continuum light for individual HII regions in LSB galaxies with multiple HII regions.

The strongest correlation was found between the line and continuum fluxes of the individual HII regions; for more intense line emission the red continuum is also more intense. A linear regression between these variables indicates a correlation coefficient of 0.62 . The same calculation for the sample of 17 BCDs in the VC leads to a correlation coefficient of 0.82 (Heller et al. 1998). The result is reminiscent of the correlation found between the SFR and the mean blue surface brightness of late-type dwarf galaxies (Brosch et al. 1998b). We conclude that the star formation of most LSB dwarf galaxies in VC depends on internal processes; a self regulating heating-cooling mechanism modulated by the local volume density of stars has to be at work, probably limiting the SFR of these types of galaxies.

In order to constraint the star formation histories we analyzed the surface color distribution of the galaxies. At present we applied this method to a single object from our sample. We mapped the color indices (U-R), (U-B) and (B-V) for VCC826 and we used the monochromatic $\mathrm{H} \alpha$-off magnitude as $\mathrm{R}$. We find that the area under the HII region is bluer than the rest of the galaxy. This indicates that there are many young stars under the HII region, and it is not just a lop-sided IMF which creates only massive stars. Therefore a truncated IMF with only stars with mass higher than $10 \mathrm{M}_{\odot}$ cannot be considered a possible explanation for the low luminosity of LSB galaxies. The color difference between 

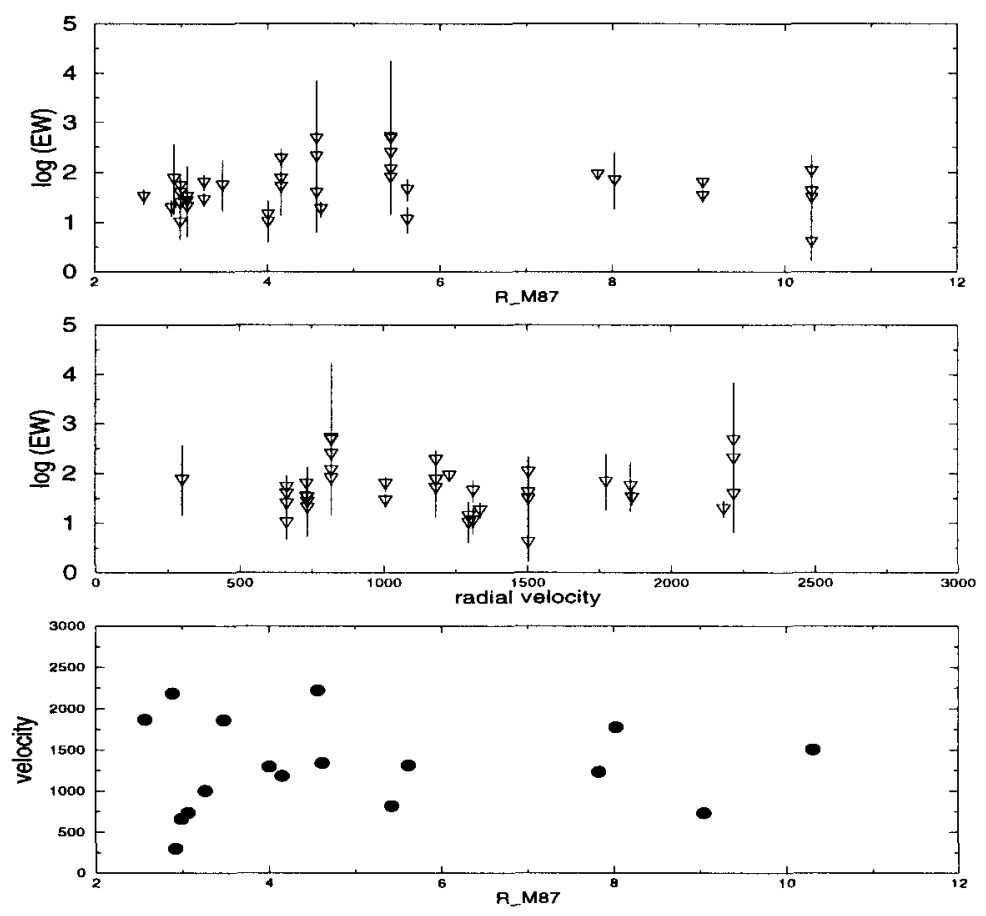

Figure 4. $\log (\mathrm{EW})$ vs. the projected angular distance in degrees from cluster center $\left(\mathrm{R}_{M 87}\right)$ (up). $\mathrm{Log}(\mathrm{EW})$ vs. heliocentric radial velocity in $\mathrm{km} / \mathrm{sec}\left(\mathrm{v}_{\odot}\right)$ (middle). Heliocentric radial velocity $\left(\mathrm{v}_{\odot}\right)$ vs. $\mathrm{R}_{M 87}$ (down).

the area under the strong HII region of VCC826 and the rest of the galaxy is only $\delta(\mathrm{U}-\mathrm{R}) \sim 0.8$; this indicates that the stellar population of the rest of the galaxy cannot be very old. Although we have not yet attempted a full evolutionary model calculation, possible combination which yield this color difference could have typically B3V stars under the HII regions and A0V in the rest of the galaxy, or $\mathrm{O} 6 \mathrm{~V}$ under the HII regions and $\mathrm{B} 7 \mathrm{~V}$ in the rest of the galaxy. This is similar to the interpretation of broad-band colors for BCDs as due to at least two stellar populations, reached by Almoznino (1996). Note that we cannot rule out the existence of even older stellar generations on the basis of the present results.

\section{Conclusions}

We found no strong evidence for environmental influences in the SF activity of LSB dwarf galaxies in the Virgo Cluster. The SF process seems to be mostly local and regulated by the local population of stars. The SF histories of LSB dwarfs and BCD are probably similar but the bursts differ in intensity. The method of local color analysis proves to be very significant in constraining possible evolutionary scenarios. 
Acknowledgments. AH acknowledges support from the US-Israel Binational Science Foundation and thanks Liese Van Zee for kindly providing comparison images. EA is supported by a special grant from the Ministry of Science and the Arts to develop TAUVEX, a UV space imaging experiment. NB is grateful for the continued support of the Austrian Friends of Tel Aviv University. Astronomical research at Tel Aviv University is partly supported by a Center of Excellence Award from the Israel Academy of Sciences.

\section{References}

Almoznino, E. 1996, PhD thesis, Tel Aviv University.

Babul, A. \& Rees, M.J. 1992, MNRAS, 255, 346.

Binggeli, B., Sandage, A. \& Tammann, G.A. 1985, AJ, 90, 1681.

Binggeli, B., Popescu, C.C. \& Tammann, G.A. 1993, A\&AS, 98, 275.

Bothun, G.D., Mould, J.R , Wirth, A. \& Caldwell, N. 1985, ApJ, 90, 697.

Brosch, N., Heller, A.B. \& Almoznino, E. 1998a, MNRAS, in press.

Brosch, N., Heller, A.B. \& Almoznino, E. 1998b, ApJ, 504, 720.

Elmegreen, B.G. 1998, in "Origins of Galaxies, Star, Planets and Life" (C. E.

Woodward, H. A. Thronson \& M. Shull, eds.), ASP series, in press.

Ferguson, H. \& Binggeli, B. 1994, A\&A Rev, 6, 67.

Hashimoto, Y., Oemler, A., Lin, H. \& Tucker, D.L. 1998, ApJ, 499, 589.

Heller, A.B., Almoznino, E. \& Brosch, N. 1988, MNRAS, in press.

Hoffman, G.L., Helou, G., Salpeter, E.E., Glosson, J. \& Sandage, A. 1987, ApJS, $63,247$.

Hoffman, G.L., Helou, G., Salpeter, E.E. \& Lewis, B. M. 1989, ApJ, 339, 812.

Hoffman, G.L., Salpeter, E.E., Farhat, B., Roos, T., Williams, H. \& Helou, G. 1996, ApJS, 105, 269.

Kennicutt, R.C., Tamblyn, P. \& Congdon, C.W. 1994, ApJ, 435, 22.

Koopmann, R.A. 1997, PhD thesis, Yale University.

Landolt, A.U. 1973, A. J 78, 958.

Landolt, A.U. 1992, A. J 104, 340.

Salpeter, E.E. \& Hoffman, G.L. 1996, ApJ, 465, 5958.

Shields, G. A., Skillman, E.D., Kennicutt, R.C. \& Zaritsky, D. 1995, RMexAA, 3,149 .

Skillman, E.D, Bothun, G.D., Murray, M.A. \& Warmels, R.H. 1987, A\&A, 185, 61 .

Tully, R. B. \& Shaya, E. 1984, ApJ, 281, 31.

van Zee, L. \& Haynes, M.P. 1998, private communication. 


\section{Searching for LSB - V}

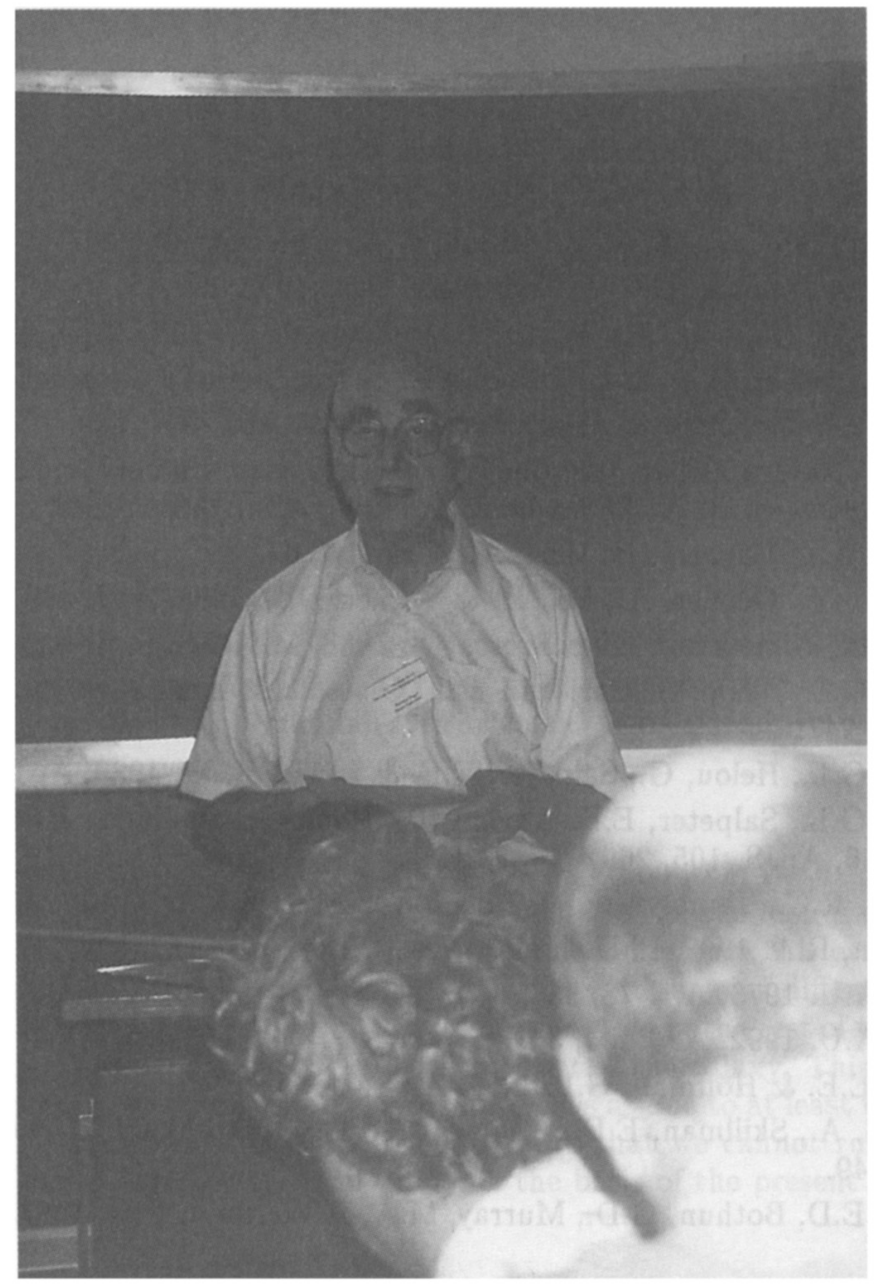

It's much further away than that, I have seen it in a vision. 